\section{Armee-Reform XXI}

\author{
Vernehmlassung zum Armeeleitbild und zur Revision \\ der Militärgesetzgebung
}

Eidgenössisches Departement

für Verteidigung, Bevölkerungsschutz

und Sport VBS

3003 Bern

Bern, den 23. Juli 2001

\section{Sehr geehrte Damen und Herren}

Wir danken Ihnen für den Einbezug der FMH in das Vernehmlassungsverfahren. Wir beschränken unsere Antwort auf zwei zentrale Anliegen der Ärzteschaft.

\section{Ausbildung, Einsatz und Dienstleistungspflicht des Militärarztes}

In den vergangenen Jahren sind Dienst und Karriere des Militärarztes zunehmend unattraktiv geworden. Hauptgründe sind die Benachteiligung der Dienstleistenden gegenüber nicht Dienstpflichtigen während des Studiums und während der Weiterbildung, und generell die Verschlechterung der Rahmenbedingungen für militärdienstleistende Ärzte sowohl im Angestelltenverhältnis - analog zur Entwicklung in der Wirtschaft - als auch in der freiberuflichen Praxis hier im Zusammenhang mit dem wachsenden Konkurrenzdruck. Zudem steht die Qualität der Ausbildung zum Militärarzt fachlich-medizinisch nicht auf der Höhe, die einen Anwärter übermässig für die militärische Weiterausbildung motivieren würde.

Weder im Armeeleitbild noch in den angestrebten Gesetzes- und Verordnungsänderungen finden wir Ansätze, diese Probleme zu lösen, eher im Gegenteil. Wir formulieren deshalb unsere Anliegen und Positionen wie folgt:

\subsection{Ausbildung}

Die Ausbildung soll ein fachliches Niveau aufweisen, welches deren ganze oder teilweise Anerkennung bzw. Anrechnung für den Erwerb eines Facharzttitels ermöglicht. Dasselbe gilt für militärische Fachdienstkurse, welche bei entsprechendem Niveau im Rahmen der FMH-Fortbildungsordnung berücksichtigt werden könnten. Die FMH, bzw. ihre in Weiter- und Fortbildungsfragen zuständigen Fachgesellschaften, bieten der Armee an dieser Stelle ausdrücklich ihre tatkräftige Mithilfe bei der Umsetzung dieser Vorgaben an. Nicht allzu fernes Ziel dieser Bemühungen muss unseres Erachtens der Aufbau eines (sinnvollerweise gemeinsamen militärischen und zivilen) Zentrums für Kriegs-, Katastrophen- und Notfallmedizin sein, wo ein speziell qualifizierter Typus Militärarzt (mit Blick auf Einsatz in in- und ausländischen Katastrophenlagen sowie friedenserhaltenden Aktionen im Aus- land), aber auch der «normale» Militärarzt und zivile Notarzt gezielt und auf hohem Niveau aus- und weitergebildet werden können.

Im übrigen verweisen wir ausdrücklich auf die detaillierten Vorschläge der Arbeitsgruppe Armee der Schweizerischen Medizinischen Interfakultätskommission (SMIFK), welche wir integral unterstützen.

Es liegt auf der Hand, dass die Ausbildung zum Militärarzt mit diesen Massnahmen deutlich an Attraktivität gewinnen und damit die Rekrutierungsbasis verbreitert werden könnte.

\subsection{Verwendung und Dienstleistungspflicht}

Seit jeher leisten Militärärzte insbesondere im Rang von SubOf, zusammen mit anderen Spezialisten, nicht nur länger Dienst als SubOf anderer Truppengattungen, sondern können im Rahmen der Übergangsrechts auch nach 42 Jahren - wenn andere SubOf ihre Militärdienstpflicht erfüllt haben - zu maximal weiteren 21 Diensttagen aufgeboten werden. Diese Dienstleistungen müssen von Militärärzten regelmässig dann erbracht werden, wenn sie sich in freier Praxis niedergelassen haben oder eine weiterführende Spitalkarriere anstreben. Die Vorlage zur Revision des Militärgesetzes hält an diesem System fest, abgesehen von einer minimalen Reduktion der Altersgrenze von 52 auf 50 Jahre. Die eingangs geschilderte Veränderung des wirtschaftlichen und konkurrenziellen Umfeldes für die Ärzteschaft zwingt uns, diese Perpetuierung der Sonderstellung von Militärärzten abzulehnen: Ärzte sollen künftig grundsätzlich gleich wie alle anderen Schweizer Bürger behandelt werden.

Wir übersehen auf der anderen Seite nicht, dass bei einer vollständigen Gleichbehandlung Militärärzte zu einem Zeitpunkt nicht oder nicht mehr in genügendem Ausmasse zur Verfügung stehen, in dem sie mit Erwerb eines Facharzttitels die auch für die Armee wünschbare medizinische Qualifikation erreicht haben.

Um den Forderungen der Ärzteschaft ebenso genüge zu tun wie den berechtigten Anliegen der Armee, skizzieren wir Ihnen folgenden Lösungsvorschlag:

a) Militärärzte leisten die gleiche Anzahl Diensttage wie andere Angehörige der Armee mit gleichem Dienstgrad.

b) Hingegen ist die Ärzteschaft bereit, mit Blick auf die Qualität der Versorgung eine höhere Altersgrenze für die Militärdienstpflicht zu akzeptieren, allerdings nur bis max. 45 Jahre. Im Gegenzug sollen Einsatz und Zuteilung der Militärärzte über einen Pool organisiert werden, mit einer garantierten maximalen Dienstleistungspflicht pro Jahr und der Möglichkeit, den Dienst aus beruflichen Gründen in Blöcken von 1 bis 5 Tagen zu leisten. Dieser Pool ist nicht von branchenfremden «Logistikern" zu betreiben, sondern soll in der Hand von (eigenständigen) Sanitätstruppen liegen (siehe dazu unten Ziff. 2).

c) Einem allfälligen Mangel an Militärärzten - trotz massiver Reduktion der Mannschaftsbestände der Armee - ist wie folgt zu begegnen: 
aa) Die Pool-Militärärzte werden in erster Linie für kriegs- bzw. militärmedizinisch qualifizierte Aufgaben eingesetzt.

bb) Für weitere Aufgaben, insbesondere die medizinische Betreuung der Truppe im Ausbildungsdienst, soll auf der Basis von privatrechtlichen Verträgen mit zivilen Ärzten (und Spitälern) zusammengearbeitet werden. Dabei drängt sich in erster Linie ein Zusammengehen mit dem sich im Aufbau befindlichen nationalen Notarztnetz auf, womit sich sowohl für die Versorgungsqualität als auch in organisatorischer Hinsicht optimale Synergien für beide öffentlichen Aufgaben, ziviles Notarztnetz und Armee, ergeben würden (Beilage).

\subsection{Fachoffiziere}

Der "flächendeckenden" Einführung von sogenannten Fachoffizieren im Sanitätsdienst stehen wir eher skeptisch gegenüber. Hingegen soll die Ernennung von Fachoffizieren, wie dies heute schon der Fall ist, für spezielle Einsätze von Fachspezialisten, insbesondere in der Ausbildung, grundsätzlich möglich sein, ja ist ausdrücklich erwünscht.

\section{Sanität: Eigenständige Truppengattung unter zentraler Führung}

In der Armee XXI soll die Sanität als eigenständige Truppengattung sang- und klanglos abgeschafft und als "Logistikprozess" (Leitbild, S. 34f) neben anderen in den neu geschaffenen Logistiktruppen aufgehen.

Wir verhehlen Ihnen nicht, dass diese Absicht in weiten Kreisen der Ärzteschaft, insbesondere bei militärisch überdurchschnittlich engagierten Ärzten, Bestürzung, um nicht zu sagen Entrüstung, hervorgerufen hat.

- "Der Mensch steht im Vordergrund" (Leitbild, S. 20). Bei allem Respekt für die Leistungen im Bereich von Nach- und Rückschub, Transport und Infrastruktur: Die Sanität geht nicht mit Waren um, sondern mit Menschen, mit Patienten. Sie sind es, die im SanD im Vordergrund stehen. Sanität ist deshalb nicht ein blosses Logistikproblem, und kann nicht auf die Frage reduziert werden, wie man am zweckmässigsten ein Objekt von A nach B verschiebt. Menschen, Patienten haben andere Bedürfnisse und brauchen ein anderes "Handling» als Verpflegung, Munition, Ersatzteile oder Betriebsstoff. Wenn Material, Nach- und Rückschub sowie Instandhaltung stets vor der individual-medizinischen Betreuung des Menschen genannt werden, sind die berechtigten Anliegen des modernen Sanitätsdienstes nicht oder zu wenig erkannt und anerkannt.

- "Der Mensch steht im Vordergrund» erfordert vielmehr die ungeteilte Verantwortung im Sanitätsdienst. Der SanD der Armee XXI muss die Effektivität und Professionalität entwickeln, um solange als möglich die individual-medizinische
Versorgung der AdA auf zivilem Niveau aufrecht erhalten zu können. Dies bedingt eine weitere Förderung des koordinierten SanD: Der SanD Armee soll subsidiär das bestehende Gesundheitswesen verstärken, um die medizinische Versorgung der Armee glaubwürdig zu gewährleisten. Um aber auch in einer ausserordentlichen Lage effizient mit 26 kantonal verschiedenen Gesundheitssystemen $\mathrm{zu}$ kooperieren, braucht es eine zentrale Steuerung des Armee SanD durch eine fachkompetente und eigenständige Stelle mit entsprechender hierarchischer Einstufung.

- Aus kriegsvölkerrechtlicher Sicht bitten wir Sie dringend $\mathrm{zu}$ prüfen, ob die geplante Neuorganisation überhaupt mit dem Genfer Abkommen von 1949 ("Rotkreuzschutz») vereinbar ist. Ergeben sich im übrigen nicht allenfalls Schwierigkeiten im Rahmen der internationalen Kooperation?

- Die Armee profitiert von der zivilen Ausbildung und dem Know-how der Militärärzte. In einem Milizsystem ist das sinnvoll und selbstverständlich. Ärzte leisteten und leisten indes im Vergleich zu den meisten Staatsbürgern überdurchschnittlich viel für die Armee, sowohl in Bezug auf die Bereitschaft (oder faktischen Verpflichtung) zur militärischen Weiterausbildung als auch bezüglich Anzahl Diensttage sowie Dauer der Dienstleistungspflicht, und sie werden dies in beschränktem Rahmen weiterhin tun (müssen). Keine zivile Berufsgattung, die ebenfalls Spezialistenwissen in die Armee einbringt, dürfte zahlen- und anteilsmässig derart breit davon betroffen sein wie die Ärzteschaft. Wir erachten es deshalb nicht als vermessen, auf der anderen Seite auch einen Ansprechpartner zu haben, der als Oberfeldarzt im Rang eines Divisionärs über eine eigenständige Stellung und über ein angemessenes Gewicht innerhalb der Armeehierarchie verfügt.

- Wie oben (Ziff. 1) dargelegt, wird nur eine entscheidende qualitative Verbesserung der Ausbildung der Militärärzte sowie eine gewisse Flexibilität bei ihrer Verwendung durch die Armee entscheidend dafür sein, ob für die Armee XXI genügend und v.a. gut qualifizierte Ärzte gewonnen werden können. Beides, die Ausbildung und der flexible Einsatz gehören in die Hand von Ärzten unter einem ärztlichen Chef.

Wir lehnen deshalb die im Entwurf vorgeschlagene Organisation des Sanitätsdienstes ab und schlagen Ihnen vielmehr vor, dessen Entwicklung in die von uns skizzierte Richtung voranzutreiben. Dies bedingt eine Ergänzung von Art. 7 Abs. 2 lit. a des Entwurfs des Militärgesetzes, wo die Sanitätstruppen (wie bisher) als eigenständige Truppengattung aufzuführen sind.

Mit freundlichen Grüssen

F M H

Dr. H. H. Brunner F.-X. Deschenaux

Präsident Generalsekretär 